\title{
Diversity and Distribution of Aquatic Plants in Khordha, Odisha, India
}

\section{Bhagyeswari Behera, Kunja Bihari Satapathy*}

Department of Botany, Centurion University of Technology and Management, Odisha

Keywords: Aquatic plants, Marshy hydrophytes, Amphibious hydrophytes, Free-floating, Submerged Fixed floating hydrophytes.

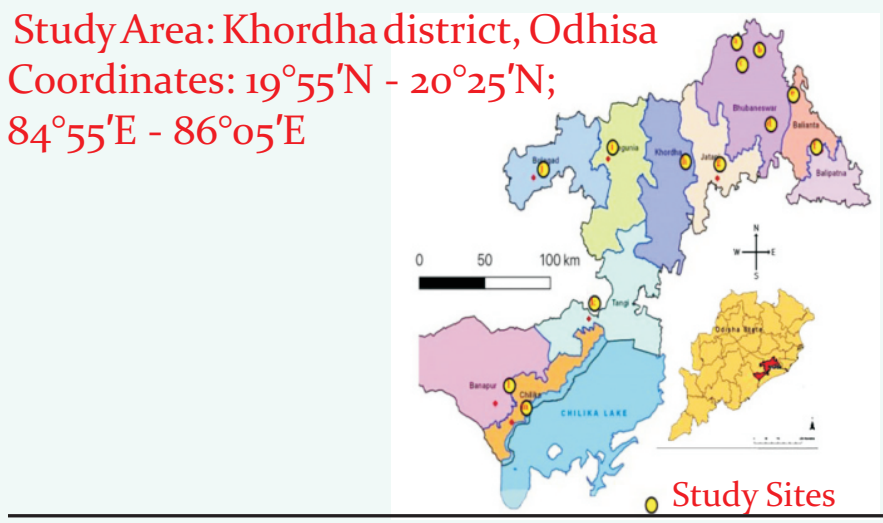

\section{Introduction:}

A plant that grows in aquatic habitat or must grow for at least a part of its life cycle in water is called an aquatic plant. Aquatic plants remain in a number of forms like submerged, free-floating, rooted floating, marshy and amphibious (Maliya, 2006). They not only support the growth of other organisms like fish, aquatic insects etc. but also play important role in ecological processes and help in maintaining the cleanliness of the water surface. Aquatic plants also play a vital role in tapping solar energy and in determining the primary productivity of the aquatic ecosystem.

Diversity and distribution of hydrophytic plants in India are well documented by several authors (Agharkar, 1923; Biswas \& Calder, 1937; Cook, 1996; Lavania et al., 1990; Hazarika \& Borthakur, 2012; Sukumaran \& Jeeva, 2011). Reports are also available on hydrophytic plants occurring in different parts of the state of Odisha (Haines, 1921-1925; Mooney, 1950; Pattnaik \& Pattanaik, 1956; Mohanty \& Choudhury, 1984; Saxena \& Brahmam, 1994-96; Subhadarsini et al., 2016); however, the present investigation is to highlight the detailed information on the diversity and distribution of aquatic plants in Khordha district of Odisha. The objective of the present investigation was to explore and map the aquatic plant diversity in the Khordha district of Odisha by exhaustive

\section{Abstract}

The results of the present survey revealed the occurrence of 211 species of hydrophytes including 200 angiosperms, 10 pteridophytes and one alga distributed in 134 genera and 61 families from 10 different localities in the study area. Besides, monocots were recorded with a maximum number of 110 species belonging to 85 genera and 16 families, whereas dicots contributed 90 species with 41 genera belonging to 37 families. Among the different categories of aquatic plants, marshy hydrophytes were represented with the maximum diversity with 131 species followed by 38 species of amphibious hydrophytes, 11 species of freefloating, 15 species of submerged and 9 species of fixed floating hydrophytes. Further taxonomic studies revealed that family Poaceae was the dominant one represented by 42 species followed by Cyperaceae with 34 species, Scrophulariaceae with 11 species, Asteraceae with 10 species, Hydrocharitaceae and Commelinaceae each with 6 species, Fabaceae with 5 species, Alismataceae, Nymphaeaceae, Polygonaceae and Onagraceae having 4 species each.

survey work. Therefore, an extensive survey of hydrophytes of the area was carried out to find out a detailed account of the diversity and distribution of hydrophytes, their habitat ecology and association, which may have economic and ecological importance.

\section{Methodology:}

Study region: Khordha district of Odisha is situated in the eastern coastal plains along the axis of the Eastern Ghat mountains. The district covers an area of $2,888 \mathrm{~km} 2$ and divided into ten C.D. Blocks namely Balianta, Balipatana, Jatni, Bhubaneswar, Tangi, Khorda sadara, Banapur, Begunia, Bolgarh and Chilika (Refer-Map).

The study was conducted during 2018-2020 involving field visits, collection of specimens, taking photographs to document the hydrophytes for their abundance and distribution. Field trips were organized in selected ten locations of the district namely Balianta, Balipatana, Jatni, Bhubaneswar, Tangi, Khorda sadara, Banapur, Begunia, Bolgarh and Chilika (Refer Map). As the flowering and fruiting seasons are different, floristic survey of water bodies and other wetlands were conducted at regular intervals to gather information on different stages of development of the aquatic specimens. Plant specimens were collected in flowering and fruiting condition as the reproductive characters are essential to ascertain the exact 
taxonomic identification of the species. The information on the distribution pattern, growth form and habitat type for all the collected specimens were recorded. The voucher specimens were identified by consulting the regional 'Flora Books' including 'The Botany of Bihar and Orissa' (Haines, 1921-1925), Supplement to the Botany of Bihar and Orissa (Mooney, 1950) and 'The Flora of Orissa' (Saxena \& Brahmam, 1994-96) and other relevant literature. Plant specimens were preserved following standard procedure and deposited in the Department of Botany, Centurion University of Technology and Management, Bhubaneswar, Odisha.

\section{Findings:}

In the present study, 211 species were recorded belonging to 134 genera of 61 families from different locations of Khordha district, Odisha. Out of these 90 species were dicots belonging to 41 genera and 37 families, whereas monocots contributed 110 species with 85 genera belonging to 16 families (Table-1 \& 2). Also, 10 species are identified as Pteridophytes belonging to 7 genera and 7 families. Of the total observed species, 139 species were recorded as marshy hydrophytes, 37 species as amphibious hydrophytes, 11 species as free-floating, 9 species as fixed floating and 15 species as submerged hydrophytes (Fig.-1) The angiosperms family Poaceae is considered as the dominant one in the study area representing with 42 species followed by Cyperaceae (34 species), Scrophulariaceae (11 species), Asteraceae (10 species), Hydrocharitaceae and Commelinaceae each one is having 6 species, Fabaceae ( 5 species), Alismataceae, Nymphaeaceae, Polygonaceae and Onagraceae having 4 species each (Fig.-3). The abundance and richness of the species in these areas were found to be varied with the change in seasons. Out of 211 species, the maximum number of plant species (124 species) were observed and specimens were collected during July to September and 71 species during the summer season (April to June), while most of the marshy species grew during the summer season in a low depth swamp area. Three insectivorous plants such as Utricularia stellaris, U. aurea and Drosera burmanii were also recorded from the area. It is interesting to note that over 50 hydrophytic plant species were found to be used by the native herbal healers of the region to treat different human ailments (Table-1).

Table-1: : Lists of aquatic plants occurring in Khordha of Odisha

\begin{tabular}{|c|c|c|c|c|}
\hline$\#$ & Scientific Name & Family & Habitat & Disease cure \\
\hline 1. & Adenostemma lavenia & Asteraceae & Amphibious & - \\
\hline 2. & Aeschynomene aspera & Fabaceae & Amphibious & Leucoderm \\
\hline 3. & Aeschynomene indica & Fabaceae & Amphibious & - \\
\hline 4. & $\begin{array}{l}\text { Alisma plantago- } \\
\text { aquatica }\end{array}$ & Alismataceae & Amphibious & - \\
\hline 5 . & Ammania multiflora & Lythraceae & Marshy & - \\
\hline 6. & Alternanthera philoxeroides & Amaranthaceae & Amphibious & - \\
\hline 7. & Alternanthera sessilis & Amaranthaceae & Marsy & $\begin{array}{l}\text { Galactorrhoea } \\
\text { Nightblindness }\end{array}$ \\
\hline 8. & Ammannia baccifera & Lythraceae & Mars & Ringworm \\
\hline & Ampelopteris prolifera & Thelypteridaceae & Amphibious & Headache \\
\hline 10. & Anagallis arvensis & Primulaceae & Amphibious & - \\
\hline
\end{tabular}

\begin{tabular}{|c|c|c|c|}
\hline 11. Aponogeton natans & Aponogetonaceae & Submerged & Hair fall \\
\hline 12. Arundo donax & Poaceae & Marshy & - \\
\hline 13. Azolla microphylla & Azollaceae & Free floating & - \\
\hline 14. Azolla pinnata & Azollaceae & Free floating & $\begin{array}{l}\text { Lactation in } \\
\text { cattle }\end{array}$ \\
\hline 15. Bacopa monnieri & Scrophulariaceae & Marshy & $\begin{array}{l}\text { Epilepsy, } \\
\text { Stammering }\end{array}$ \\
\hline 16. Bergia ammannioides & Elatinaceae & Marshy & Fungal infect. \\
\hline 17. Bergia capensis & Elatinaceae & Amphibious & - \\
\hline 18. Blyxa echinosperma & Hydrocharitaceae & Submerged & Ear ache \\
\hline 19. Brachiaria distachya & Poaceae & Marshy & - \\
\hline 20. Brachiaria milliformis & Poaceae & Marshy & - \\
\hline 21. Brachiaria mutica & Poaceae & Marshy & $\begin{array}{l}\text { Diarrhoea, } \\
\text { worm infect. }\end{array}$ \\
\hline 22. Bulbostylis densa & Cyperaceae & Marshy & - \\
\hline 23. Caesulia axillaris & Asteraceae & Marshy & Cut wounds \\
\hline 24. Canscora diffusa & Gentianaceae & Marshy/Wetland & $1-$ \\
\hline 25. Centipeda minima & Asteraceae & Moist places & - \\
\hline 26. Centella asiatica & Apiaceae & Marshy & $\begin{array}{l}\text { Stomach disor } \\
\text { der, Hair grow }\end{array}$ \\
\hline 27. Ceratophyllum demersum & Ceratophyllaceae & Submerged & Glycosuria \\
\hline 28. Ceratopteris thalictroides & Parkeriaceae & Marshy & Skin diseases \\
\hline 29. Chara vulgaris & Characeae & Submerged & - \\
\hline 30. Chloris barbata & Poaceae & Marshy & - \\
\hline 31. Coix lacryma-jobi & Poaceae & Marshy & Jaundice \\
\hline 32. Coldenia procumbens & Boraginaceae & Marshy/Wetland & $1-$ \\
\hline 33. Colocasia esculenta & Araceae & Amphibious & - \\
\hline 34. Commelina benghalensis & Commelinaceae & Marshy & - \\
\hline 35. Commelina erecta & Commelinaceae & Marshy & - \\
\hline 36. Commelina paludosa & Commelinaceae & Marshy & - \\
\hline 37. Crinum defixum & Amaryllidaceae & Amphibious & Earache, Dysuria \\
\hline 38. Cyanotis cristata & Commelinaceae & Marshy & - \\
\hline 39. Cyperus articulatus & Cyperaceae & Marshy & - \\
\hline 40. Cyperus brevifolius & Cyperaceae & Marshy & - \\
\hline 41. Cyperus difformis & Cyperaceae & Marshy & - \\
\hline 42. Cyperus distans & Cyperaceae & Marshy & - \\
\hline 43. Cyperus flabelliformis & Cyperaceae & Marshy & - \\
\hline 44. Cyperus halpan & Cyperaceae & Marshy & - \\
\hline 45. Cyperus haspan Juncoides & Cyperaceae & Marshy & - \\
\hline 46. Cyperus imbricatus & Cyperaceae & Marshy & - \\
\hline 47. Cyperus iria & Cyperaceae & Marshy & - \\
\hline 48. Cyperus kyllingia & Cyperaceae & Marshy & - \\
\hline 49. Cyperus paniceus & Cyperaceae & Marshy & - \\
\hline 50. Cyperus pilosus & Cyperaceae & Marshy & - \\
\hline 51. Cyperus polystachyos & Cyperaceae & Marshy & - \\
\hline 52. Cyperus puncticulatus & Cyperaceae & Marshy & - \\
\hline 53. Cyperus pygmaeus & Cyperaceae & Muddy swamps & - \\
\hline 54. Cyperus rotundus tuberosus & Cyperaceae & Marshy & $\begin{array}{l}\text { Diarrhoea, } \\
\text { Irregular mens. }\end{array}$ \\
\hline 55. Cyperus triceps & Cyperaceae & Marshy & - \\
\hline 56. Cyrtococcum trigonum & Poaceae & Marshy & - \\
\hline 57. Dentella repens & Rubiaceae & Marshy & - \\
\hline 58. Dopatrium junceum & Scrophulariaceae & Marshy & - \\
\hline 59. Drosera burmannii & Droseraceae & Marshy & - \\
\hline 6o. Echinochloa colona & Poaceae & Marshy & - \\
\hline 61. Echinochloa crusgalli & Poaceae & Marshy & - \\
\hline 62. Echinochloa stagnina & Poaceae & Amphibious & Diabetes \\
\hline 63. Eclipta prostrata & Asteraceae & Marshy & $\begin{array}{l}\text { Premature } \\
\text { graying of hair }\end{array}$ \\
\hline 64. Eichhornia crassipes & Pontederiaceae & Free floating & - \\
\hline 65. Eleocharis dulcis & Cyperaceae & Marshy & - \\
\hline 66. Elytrophorus spicatus & Poaceae & Amphibious & - \\
\hline 67. Emilia sonchifolia & Asteraceae & Marshy & - \\
\hline 68. Enydra fluctuans & Asteraceae & Amphibious & Hair fall \\
\hline 69. Eragrostis ciliaris & Poaceae & Marshy & $\begin{array}{l}\text { Diarrhoea, } \\
\text { dysentery }\end{array}$ \\
\hline 70. Eragrostis japonica & Poaceae & Marshy & Headache \\
\hline 71. Eragrostis pilosa & Poaceae & Marshy & - \\
\hline 72. Eragrostis uniloides & Poaceae & Marshy & - \\
\hline 73. Eriocaulon quinquangulare & Eriocaulaceae & Amphibious & Jaundice \\
\hline 74. Eriochloa procera & Poaceae & Swamps & - \\
\hline 75. Euryale ferox & Nymphaeaceae & Fixed floating & - \\
\hline 76. Fimbristylis aestivalis & Cyperaceae & Marshy & - \\
\hline 77. Fimbristylis argentea & Cyperaceae & Marshy & - \\
\hline 78. Fimbristylis dichotoma & Cyperaceae & Marshy & - \\
\hline 79. Fimbristylis miliacea & Cyperaceae & Marshy & - \\
\hline 8o. Fimbristylis ovata & Cyperaceae & Moist-wet places & - \\
\hline 81. Fimbristylis schoenoides & Cyperaceae & Amphibious & - \\
\hline
\end{tabular}




\begin{tabular}{|c|c|c|c|}
\hline \multirow{2}{*}{ 82. Fuirena umbellata } & \multirow[b]{2}{*}{ Cyperaceae } & \multicolumn{2}{|c|}{ (Marshy, some times floating) } \\
\hline & & Amphibious & - \\
\hline 83. Glinus lotoides & Molluginaceae & Marshy & - \\
\hline 84. Glinus oppositifolius & Molluginaceae & Marshy & - \\
\hline 85. Gnaphalium polycaulon & Asteraceae & Marshy & - \\
\hline 86. Hackelochloa granularis & Poaceae & Marshy & - \\
\hline 87. Hedyotis diffusa & Rubiaceae & Marshy & - \\
\hline 88. Heliotropium strigosum & Boraginaceae & Marshy & - \\
\hline 89. Hemarthria compressa & Poaceae & Marshy & - \\
\hline 9o. Hemionitis arifolia & \multicolumn{2}{|c|}{ Hemionitidaceae Moist locality } & - \\
\hline 91. Hydrilla verticillata & Hydrocharitaceae & Submerged & Wound \\
\hline 92. Hydrocera triflora & Balsaminaceae & Amphibious & - \\
\hline 93. Hydrocotyle sibthorpioides & Apiaceae & Marshy & - \\
\hline 94. Hydrolea zeylanica & Hydroleaceae & Amphibious & Skin injury \\
\hline 95. Hygrophila polysperma & Acanthaceae & Submerged & - \\
\hline 96. Hygrophila schulli & Acanthaceae & Amphibious & - \\
\hline 97. Hygroryza aristata & Poaceae & Free floating & Urinary infec. \\
\hline 98. Hymenachne acutigluma & Poaceae & Amphibious & - \\
\hline 99. Ipomoea aquatica & Convolvulaceae & Fixed floating & - \\
\hline 10o.Ipomoea carnea & Convolvulaceae & Marshy & - \\
\hline 101. Isachne miliacea & Poaceae & Marshy & - \\
\hline 102. Ischaemum indicum & Poaceae & Marshy & - \\
\hline 103. Ischaemum rugosum & Poaceae & Wet lands & - \\
\hline 104.Isleima prostratum & Poaceae & Marshy & - \\
\hline 105. Kyllinga brevifolia & Cyperaceae & Marshy & - \\
\hline 106.Kyllinga nemolaris & Cyperaceae & Marshy & - \\
\hline 107. Kyllinga triceps & Cyperaceae & Marshy & - \\
\hline 108.Leersia hexandra & Poaceae & Amphibious & - \\
\hline 109.Lemna perpusilla & Lemnaceae & Free floating & Body ache \\
\hline 110. Limnophila heterophylla & Scrophulariaceae & Amphibious & Dyspepsia \\
\hline 111. Limnophila indica & Scrophulariaceae & Amphibious & - \\
\hline 112. Limnophila repens & Scrophulariaceae & Marshy & - \\
\hline 113. Limnophyton obtusifolium & Alismataceae & Marshy & Dysentery \\
\hline 114. Lindernia anagallis & Scrophulariaceae & Marshy & - \\
\hline 115. Lindernia antipoda & Scrophulariaceae & Marshy & - \\
\hline 116. Lindernia crustacea & Scrophulariaceae & Marshy & - \\
\hline 117. Lindernia hyssopioides & Linderniaceae & Marshy & - \\
\hline 118. Lindernia parviflora & Schrophulariaceae & Marshy & - \\
\hline 119. Lindernia rotundifolia & Scrophulariaceae & Marshy & - \\
\hline 120.Lippia javanica & Verbenaceae & Marshy & Burning sens. \\
\hline 121. Lobelia alsinoides & Campanulaceae & Marshy & - \\
\hline 122. Lobelia heyneana & Campanulaceae & Marshy & - \\
\hline 123. Ludwigia adscendens & Onagraceae & Fixed floating & - \\
\hline 124. Ludwigia octovalvis & Onagraceae & Amphibious & $\begin{array}{l}\text { Eczema, } \\
\text { Leucorrhoea }\end{array}$ \\
\hline 125. Ludwigia perennis & Onagraceae & Marshy & - \\
\hline 126. Ludwigia prostrata & Onagraceae & Wet places & - \\
\hline 127. Mariscus compactus & Cyperaceae & Marshy & - \\
\hline 128. Marsilea minuta & Marsileaceae & Amphibious & $\begin{array}{l}\text { Epilepsy, } \\
\text { Insomnia }\end{array}$ \\
\hline 129. Marsilea quadrifolia L. & Marsileaceae & Amphibious & - \\
\hline 130.Mazus pumilus & Scrophulariaceae & Marshy & - \\
\hline 131. Melastoma malabathricum & Melastomaceae & Marshy/Wet pl. & - \\
\hline 132. Melinis repens & Poaceae & Marshy & - \\
\hline 133. Melochia corchorifolia & Sterculiaceae & Amphibious & - \\
\hline 134. Mnesithea laevis & Poaceae & Marshy & - \\
\hline 135. Monochoria hastata & Pontederiaceae & Amphibious & - \\
\hline 136. Monochoria vaginalis & Pontederiaceae & Amphibious & $\begin{array}{l}\text { Asthma, } \\
\text { Toothache }\end{array}$ \\
\hline 137. Murdannia vaginata & Commelinaceae & Marshy & - \\
\hline 138. Myriophyllum tetrandrum & Haloragaceae & Submerged & - \\
\hline 139. Najas graminea & Hydrocharitaceae & Submerged & Dysentery \\
\hline 140.Nechamandra alternifolia & Hydrocharitaceae & Submerged & - \\
\hline 141. Nelumbo nucifera & Nymphaeaceae & Fixed floating & - \\
\hline 142. Neptunia oleracea & Mimosaceae & Floating & Earache \\
\hline 143. Nicotiana plumbaginifolia & Solanaceae & Marshy & Piles \\
\hline 144. Nymphaea nouchali & Nymphaeaceae & Fixed Floationg & - \\
\hline 145. Nymphaea pubescens & $\begin{array}{l}\text { Nymphaeaceae } \\
\text { Dysmenorrhoea }\end{array}$ & Fixed floating & \\
\hline 146.Nymphoides hydrophylla & Menyanthaceae & Fixed floating & - \\
\hline 147. Nymphoides indica & Menyanthaceae & Fixed floating & Ulcers on scalp \\
\hline 148.Oplismenus burmanii & Poaceae & Marshy & - \\
\hline 149. Oplismenus compositus & Poaceae & Marshy & - \\
\hline 15o. Oryza rufipogon & Poaceae & Marshy & Stomachache \\
\hline 151. Ottelia alismoides & Hydrocharitaceae & Submerged & Diarrhoea \\
\hline 152. Oxalis corniculata & Oxalidaceae & Marshy & $\begin{array}{l}\text { Diarrhoea, } \\
\text { Warts }\end{array}$ \\
\hline Panicum paludosum & Poacea & Marshy & \\
\hline
\end{tabular}

\begin{tabular}{|c|c|c|c|}
\hline 154. Panicum psilopodium & Poaceae & Marshy & - \\
\hline 155. Paspalidium flavidum & Poaceae & Marshy & Skin itching \\
\hline 156. Paspalidium geminatum & Poaceae & Marshy & - \\
\hline 157. Paspalum distichum L. & Poaceae & Marshy & - \\
\hline 58. Paspalum scrobiculatum & Poaceae & Marshy & Diabetes \\
\hline 159. Pentapetes phoenicea & Sterculiaceae & Marshy & - \\
\hline 16o. Phyla nodiflora & Verbenaceae & Marshy & - \\
\hline 161. Phyllanthus urinaria & Euphorbiaceae & Marshy & - \\
\hline 162.Pilea microphylla & Urticaceae & Marshy & - \\
\hline 163. Pistia stratiotes & Araceae & Free floating & Diabetes \\
\hline 164. Polygonum barbatum & Polygonaceae & Amphibious & Stomach poison \\
\hline 165. Polygonum glabrum & Polygonaceae & Amphibious & - \\
\hline 166.Polygonum hydropiper & Polygonaceae & Amphibious & - \\
\hline 167.Polygonum plebeium & Polygonaceae & Amphibious & - \\
\hline 168. Polygonum pulchrum & Polygonaceae & Marshy & - \\
\hline 169.Portulaca quadrifolia & Portulacaceae & Marshy & - \\
\hline 170. Potamogeton nodosus & Potamogetonaceae & Submerged & Toothache \\
\hline 171. Potamogeton pusillus & Potamogetonaceae & Submerged & - \\
\hline 172. Pteris cretica & Pteridaceae & Marshy & - \\
\hline 173. Ranunculus scleratus & Ranunculaceae & Amphibious & $\begin{array}{l}\text { Eczema, } \\
\text { Bronchiti }\end{array}$ \\
\hline 174. Rikliella squarrosa & Cyperaceae & Marshy & - \\
\hline 175. Rotala indica & Lythraceae & Marshy & - \\
\hline 176.Saccharum spontaneum & Poaceae & Marshy & Dysentery \\
\hline 177. Sacciolepis indica & Poaceae & Marshy & - \\
\hline 178. Sacciolepis interrupta & Poaceae & Marshy & - \\
\hline 179. Sacciolepis mysuroides & Poaceae & Marshy & - \\
\hline 180.Sagittaria guayanensis & Alismataceae & Amphibious & - \\
\hline 181. Sagittaria trifolia & Alismataceae & Swamps & Skin infection \\
\hline 182. Salvinia cucullata & Salviniaceae & Free floating & - \\
\hline 183. Salvinia molesta & Salviniaceae & Free floating & $\begin{array}{l}\text { Gastrointestinal } \\
\text { in Cattle }\end{array}$ \\
\hline 184.Scirpus articulatus & Cyperaceae & Amphibious & Dysentery \\
\hline 185. Scirpus grossus & Cyperaceae & Amphibious & Vomiting \\
\hline 186.Scirpus lateriflorus & Cyperaceae & & - \\
\hline 187. Selaginella repanda & Selaginellaceae & Marshy/Damp & - \\
\hline 188.Sesbania javanica & Fabaceae & Marshy & - \\
\hline 189.Sesuvium portulacastrum & Aizoaceae & Marshy & - \\
\hline 190.Setaria pumila & Poaceae & Marshy & - \\
\hline 191. Smithia sensitiva & Fabaceae & Wet places & - \\
\hline 192.Sphaeranthus indicus & Asteraceae & Wet grounds & General debility \\
\hline 193. Sphenoclea zeylanica & Campanulaceae & Marshy & Jaundice \\
\hline 194. Spilanthes paniculata & Asteraceae & Marshy & Scabies \\
\hline 195. Spiranthes sinensis & Orchidaceae & Marshy & - \\
\hline 196.Spirodela polyrhiza & Lemnaceae & Free floating & Body swelling \\
\hline 197. Sporobolus indicus & Poaceae & Wetlands & - \\
\hline 198.Stachytarpheta jamaicensis & Verbenaceae & Marshy & - \\
\hline 199.Synedrella nodiflora & Asteraceae & Marshy & - \\
\hline 200.Tonningia axillaris & Commelinaceae & Marshy & - \\
\hline 201. Trapa natans & Trapaceae & Free floating & $\begin{array}{l}\text { Debility, } \\
\text { Menorrhagia }\end{array}$ \\
\hline 202.Typha angustata & Typhaceae & Amphibious & Jaundice \\
\hline 203.Typhonium trilobatum & Araceae & Marshy & - \\
\hline 204.Utricularia aurea & Utriculariaceae & Submerged & - \\
\hline 205.Utricularia stellaris & Utriculariaceae & Submerged & Eczema \\
\hline 206.Vallisneria natans & Hydrocharitaceae & Submerged & - \\
\hline 207. Vetiveria zizanioides & Poaceae & $\begin{array}{l}\text { Damp/Swampy } \\
\text { place }\end{array}$ & $\begin{array}{l}\text { Menorrhagia, } \\
\text { Dysuria }\end{array}$ \\
\hline 208.Vigna adenantha & Fabaceae & Marshy & - \\
\hline 209. Wahlenbergia marginata & Campanulaceae & Marshy & - \\
\hline 210. Wolfia globosa & Lemnaceae & Free Floating & - \\
\hline 211. Xyris indica & Xyridaceae & Marshy & - \\
\hline
\end{tabular}

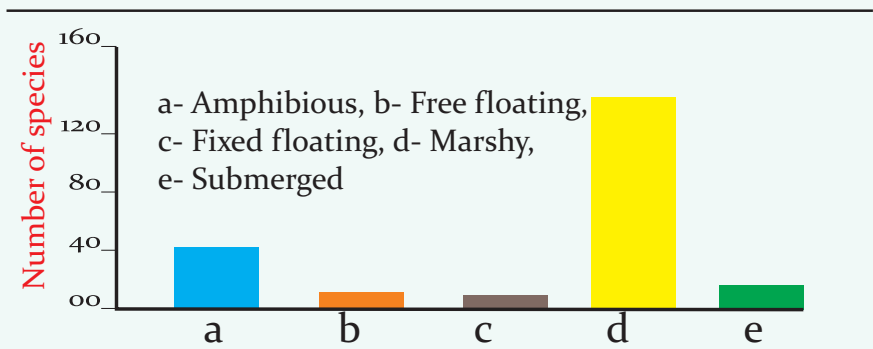

Figure -2: Habitat-wise distribution of aquatic plants in Khordha district of Odisha. 
Table 2: Distribution of aquatic plants in Khordha, Odisha

\begin{tabular}{llllll}
\hline Taxon & \multicolumn{2}{l}{ Angiosperms } & Pteridophyte & Algae & Total \\
& Dicot & Monocot & & & \\
\hline Family & 37 & 16 & 7 & 1 & 61 \\
Genera & 41 & 85 & 7 & 1 & 134 \\
Species & 90 & 110 & 10 & 1 & 211 \\
\hline
\end{tabular}

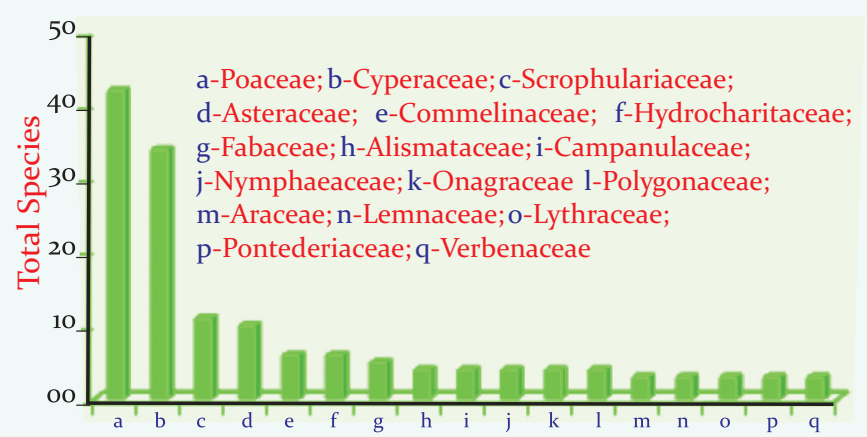

Figure-2: Family-wise distribution aquatic plants in Khordha district of Odisha

\section{Conclusion:}

The present study reveals the diversity and occurrence of 211 aquatic plant species in Khordha district of Odisha indicating the hydrophytic floral richness of the study area. The data on aquatic flora of the district will be of immense help for future taxonomic as well as environmental management related studies especially in the study sites with high anthropogenic disturbances and having maximum water pollution on account of urbanization and massive industrial activities. The study also indicates the need for taking up regional or district-wise studies for a better understanding of the distribution of hydrophytes and to develop location-specific conservation strategies using this baseline data to protect the valuable and yet poorly studied economically important plant species. It is suggested that the native hydrophytic plants with therapeutic claims need to be further investigated in view of their bioactive components so that the drug values can be put to use in modern and synthetic medicines.

\section{Acknowledgements:}

The authors are thankful to the authorities of Centurion University of Technology and Management, Odisha for providing necessary facilities during the course of this investigation..

\section{References:}

Agharkar, S.P. (1923): The present position of our knowledge of the aquatic flora of India. J. Indian Bot. Soc., 3:252-26o.

Biswas, K. \& Calder, C. (1937): Handbook of common water and marsh plants of India and Burma. Pub. by: Govt. Press, Delhi.

Cook, C.D.K. (1996): Aquatic and wetland plants of India. Pub. by: Oxford University Press, New York. pp. 385

Haines, H.H. (1921-1925): The Botany of Bihar and Odisha, Vol. 1-6 parts. Pub. by: Adlard and Sons and West Newman, Ltd., London.

Hazarika, S. \&, Borthakur, S.K. (2012): Hydrophytic flora of Assam - I: five new records. Pleione, 6(2).

Lavania, G.S., Paliwal, S.C. \& Gopal, B. (1990): Aquatic vegetation of the Indian subcontinent, pp. 29 - 76. In: (Brij Gopal ed.) Ecology and Management of Aquatic vegetation in the Indian subcontinent. Pub. by: Kluwer Academic Publishers, Dordsecht Boston/London.

Mohanty, M. \& Choudhury, B.P. (1984): Addition of Hydrophytes of Cuttack. Bull. Env. Sci., 1:9-12.

Maliya, S.D. (2006): The aquatic and wetland flora of Mainpuri district, U.P., India.J. Eco. Taxo. Bot., 30(3):533-546.

Mooney, H.F. (1950): Supplement to the Botany of Bihar and Orissa. Pub. by: Catholic Press, Ranchi.

Pattnaik, H. \& Pattanaik, N.K. (1956): The hydrophytes of Cuttack. J. Indian Bot. Soc., 35:167-170.

Saxena, H.O. \& Brahmam, M. (1994-96): The Flora of Orissa. Vol. I-IV. Pub. by: Regional Research Laboratory (CSIR), Bhubaneswar and Orissa Forest Development Corporation Ltd., Bhubaneswar.

Subhadarsini, S., Nayak, S.K. \& Satapathy, K.B. (2016): Study of floral diversity with special reference to hydrophytes in Bhubaneswar and its adjoining areas, Odisha, India. Int. Res. L.Biol. Sci., 5(9):1-6.

Sukumaran, S. \& Jeeva, S. (2011): Angiosperm flora from wetlands of Kanyakumari district, Tamilnadu. Indian J. Species list. Distribu., 7(4). 